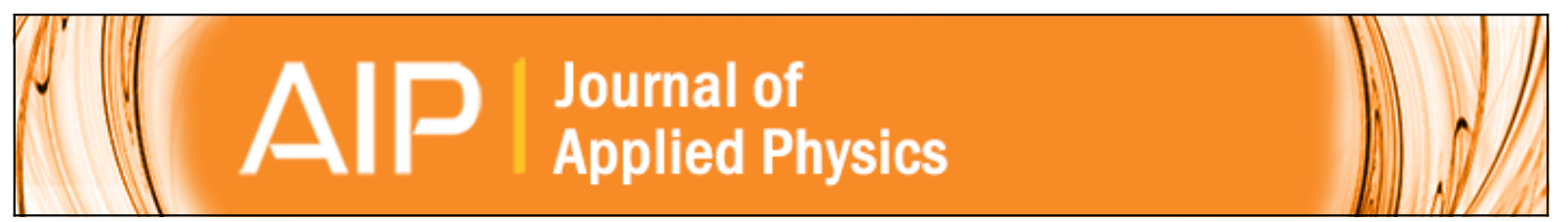

\title{
Characterization of the magnetic interactions of multiphase magnetocaloric materials using first-order reversal curve analysis
}

V. Franco, F. Béron, K. R. Pirota, M. Knobel, and M. A. Willard

Citation: Journal of Applied Physics 117, 17C124 (2015); doi: 10.1063/1.4919121

View online: http://dx.doi.org/10.1063/1.4919121

View Table of Contents: http://scitation.aip.org/content/aip/journal/jap/117/17?ver=pdfcov

Published by the AIP Publishing

\section{Articles you may be interested in}

The effect of distributed exchange parameters on magnetocaloric refrigeration capacity in amorphous and nanocomposite materials

J. Appl. Phys. 111, 07 A334 (2012); 10.1063/1.3679456

Magnetocaloric effect and critical exponents of Fe77C05.5Ni5.5Zr7B4Cu1: A detailed study

J. Appl. Phys. 109, 07A905 (2011); 10.1063/1.3535191

Optimization of the refrigerant capacity in multiphase magnetocaloric materials

Appl. Phys. Lett. 98, 102505 (2011); 10.1063/1.3560445

Influence of $\mathrm{Co}$ and $\mathrm{Ni}$ addition on the magnetocaloric effect in Fe $88-2 \times \mathrm{Co} \times \mathrm{Ni} \times \mathrm{Zr} 7 \mathrm{~B} 4 \mathrm{Cu} 1$ soft magnetic amorphous alloys

Appl. Phys. Lett. 96, 182506 (2010); 10.1063/1.3427439

The influence of Co addition on the magnetocaloric effect of Nanoperm-type amorphous alloys

J. Appl. Phys. 100, 064307 (2006); 10.1063/1.2337871

MIT LINCOLN

LABORATORY CAREERS

Discover the satisfaction of innovation and service

to the nation
- Space Control

- Air \& Missile Defense

- Communications Systems \& Cyber Security

- Intelligence, Surveillance and

Reconnaissance Systems

- Advanced
Electronics
- Tactical Systems
" Homeland
Protection
- Air Traffic Control

LINCOLN LABORATORY

MassachusetTs Institute OF TechNology

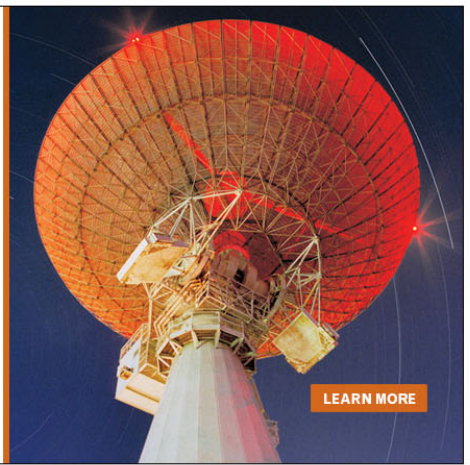




\title{
Characterization of the magnetic interactions of multiphase magnetocaloric materials using first-order reversal curve analysis
}

\author{
V. Franco, ${ }^{1,2, a)}$ F. Béron, ${ }^{2}$ K. R. Pirota, ${ }^{2}$ M. Knobel, ${ }^{2}$ and M. A. Willard ${ }^{3}$ \\ ${ }^{1}$ Dpto. Física de la Materia Condensada, ICMSE-CSIC, Universidad de Sevilla, P.O. Box 1065, 41080 Sevilla, \\ Spain \\ ${ }^{2}$ Instituto de Física Gleb Wataghin, Universidade Estadual de Campinas (UNICAMP), Campinas 13083-859, \\ São Paulo, Brazil \\ ${ }^{3}$ Department of Materials Science and Engineering, Case Western Reserve University, Cleveland, Ohio 44106, \\ USA
}

(Presented 4 November 2014; received 2 September 2014; accepted 6 January 2015; published online 28 April 2015)

\begin{abstract}
In order to understand the magnetocaloric response of materials, it is important to analyze the interactions between the different phases present in them. Recent models have analyzed the influence of these interactions on the magnetocaloric response of composites, providing an estimate value of the interaction field that is consistent with experimental results. This paper analyzes to which extent magnetization first-order reversal curve (FORC) method can be used to calculate these interactions. It is shown that the different field ranges that are explored using these techniques (inside the hysteretic region for FORC; close to magnetic saturation for magnetocaloric effect) produce interaction field values that differ in order of magnitude, with FORC being sensitive to the lower values of the interaction field and magnetocaloric analysis accounting for the larger interactions. (C) 2015 AIP Publishing LLC. [http://dx.doi.org/10.1063/1.4919121]
\end{abstract}

Magnetic refrigeration, a technology that is much more energetically efficient and environmental friendly than conventional refrigeration based on the compression and expansion of gases, is not implemented yet in commercial devices due to issues related to both the engineering design of the refrigerator and to limitations of the current magnetocaloric materials. ${ }^{1}$ These issues are interlinked, in the sense that the term "optimal material" can only be understood when associated to a particular refrigerator design. However, there are numerous attempts to find materials with improved magnetocaloric response that can help design engineers to improve or modify their devices. ${ }^{2}$

There are two alternative approaches in order to search for optimized magnetocaloric response. The first one consists in looking for new alloys and compounds, usually by tailoring the composition of known magnetocaloric materials, in order to tune their Curie temperatures $\left(T_{\mathrm{c}}\right)$, increase their magnetic entropy change $\left(\Delta \mathrm{S}_{\mathrm{M}}\right)$, and ultimately their refrigerant capacity $(R C)$. An alternative is to apply materials engineering techniques, such as controlling microstructure and developing composites, to well-known phases with good magnetocaloric response, with the aim of enhancing their properties. Multiphase magnetocaloric materials and composites are an efficient way to produce table-like magnetocaloric effect and to obtain materials with larger refrigerant capacity than their constituent phases. ${ }^{3}$ It has recently been shown that the proper selection of Curie temperatures and fraction of phases of composite materials can produce $R C$ enhancements of the order of $100 \%$ when compared to that of the pure starting phases. ${ }^{4}$ The general features of these composites can be properly described

\footnotetext{
${ }^{\text {a) }}$ Author to whom correspondence should be addressed. Electronic mail: vfranco@us.es.
}

by considering an interaction-free model. ${ }^{5}$ However, a more careful analysis that considers dipolar interactions between phases improves the agreement between experimental results and numerical predictions. ${ }^{6}$ Using this analysis, it was possible to predict an interaction field of the order of 4000 Oe between the individual phases of a composite formed by $\mathrm{Fe}_{71.5} \mathrm{Co}_{8.25} \mathrm{Ni}_{8.25} \mathrm{Zr}_{7} \mathrm{~B}_{4} \mathrm{Cu}_{1}$ and $\mathrm{Fe}_{66} \mathrm{Co}_{11} \mathrm{Ni}_{11} \mathrm{Zr}_{7} \mathrm{~B}_{4} \mathrm{Cu}_{1}$.

Magnetization first-order reversal curve (FORC) method is becoming a more usual technique for determining interactions in multiphase magnetic materials. ${ }^{7}$ Initially proposed as a method to identify the Preisach model parameters, ${ }^{8}$ it was later extended as a model-independent technique to characterize the irreversibility in magnetic materials magnetization reversal. $^{9}$

In this work, we use FORC analysis to quantify the magnitude of the interaction field in several multiphase composites and results are compared with the one obtained by the magnetocaloric analysis. Amorphous ribbons of $\mathrm{Fe}_{88-2 x} \mathrm{Co}_{x} \mathrm{Ni}_{x} \mathrm{Zr}_{7} \mathrm{~B}_{4} \mathrm{Cu}_{1}$ (typically 2-3 mm wide and $\sim 20 \mu \mathrm{m}$ thick) with composition range $x=2.75,5.5,8.25$, and 11 , were obtained by a melt-spinning technique. In the following, alloys will be denoted by their respective Co and Ni content, $x$. Further details about sample preparation, microstructural, and magnetic characterization are given elsewhere. ${ }^{10}$ Composite samples were prepared by putting two ribbons of different compositions in mechanical contact, keeping them together inside the sample holder. This resembles the method used for the measurement on the magnetocaloric response (layered combinations of pieces of the different alloys). ${ }^{4,5}$

The determination of first-order reversal curves consists in the measurement of a set of minor hysteresis curves that are measured between different reversal fields, $H_{\mathrm{r}}$, and the 
saturation field (Fig. 1). It is worth mentioning that the reversal curves will coincide with the saturation loop in two circumstances: either when the reversal field is positive and larger than the irreversibility onset, or once a negative $H_{\mathrm{r}}$ overcomes the irreversibility region of the sample (around 2 Oe for the samples studied in this paper). When these minor loops are represented as a function of the applied field, $H$, the FORC distribution $\rho$ can be calculated from the variation of magnetization $M$ as a function of the applied field and the reversal field

$$
\rho\left(H, H_{r}\right)=-\frac{1}{2} \frac{\partial^{2} M\left(H, H_{r}\right)}{\partial H_{r} \partial H} \quad\left(H \geq H_{r}\right) .
$$

This distribution is usually represented as a function of the interaction $\left(H_{u}\right)$ and coercive $\left(H_{c}\right)$ fields axes of the distribution of hysterons (i.e., square hysteresis operators).

Temperature dependent magnetization curves were measured in a Lakeshore 7407 VSM equipped with a furnace. Room temperature major hysteresis curves and FORCs of $10 \mathrm{~cm}$ long ribbons were acquired in a homemade highprecision AC hysteresis loop tracer previously adapted to FORC measurements. ${ }^{11}$ A longitudinal saturation field of 50 Oe was applied, while each FORC reversal field was separated by a field interval of 0.1 Oe. The external magnetic field is a triangular wave with variable amplitude, DC offset, and frequency during the FORC measurement, keeping constant the magnetic field sweep rate at $750 \mathrm{Oe} / \mathrm{s}$ throughout the measurements. To obtain a good signal-to-noise ratio, up to 250 curves were averaged for each FORC minor curve. To ensure a step size ratio of 2 (reversal field vs applied field step sizes), a mean value of 0.05 Oe was used for the applied field step size, yielding between 50 and 60 FORC minor curves. The software for the FORC calculation is custom made, based on a Shepard algorithm for a data bivariate interpolation on irregular grid. ${ }^{12}$ Taking into account the soft magnetic behavior of the alloys used in this study, with coercivities of the order of 0.1 Oe, temperature dependent hysteresis loops could not be measured in the VSM due to the

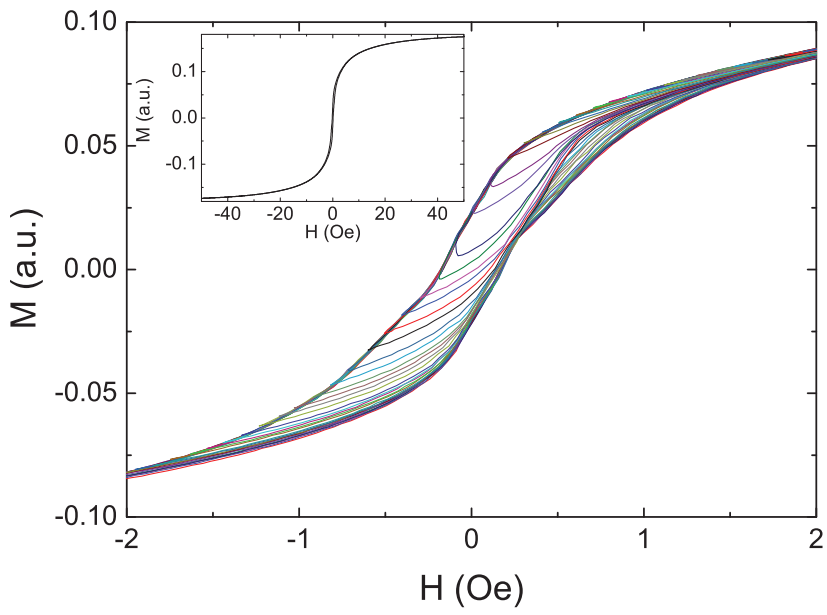

FIG. 1. Typical room temperature first-order reversal curves (main panel) and major hysteresis loop (inset) of the composite formed by alloys with $x=8.25$ and $x=11$.

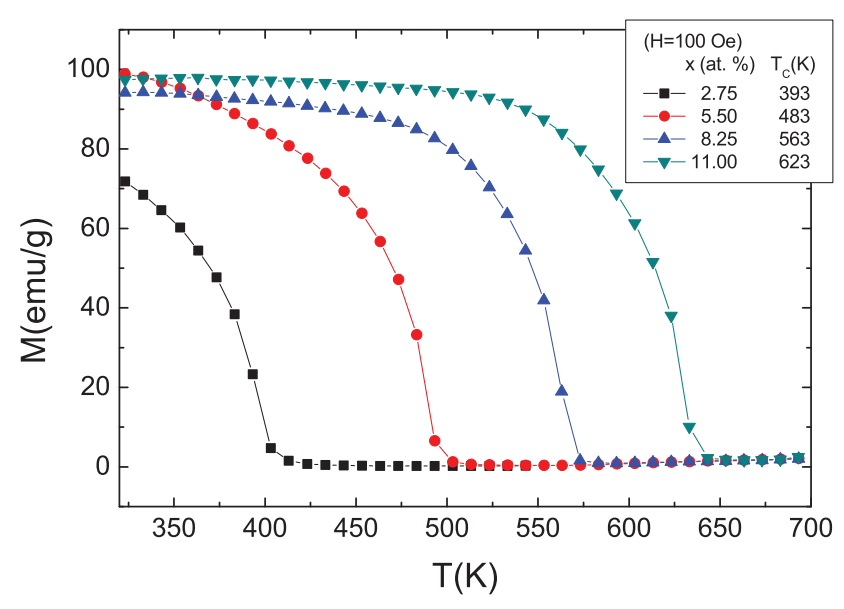

FIG. 2. Temperature dependence of the low field magnetization of the individual phases used in the composites.

uncertainty in field determination within the hysteretic region. This made us restrict the FORC analysis to room temperature.

Figure 2 shows the low field $(100 \mathrm{Oe})$ temperature dependent magnetization curves of the four studied alloys, showing the increasing value of $T_{c}$ with increasing $\mathrm{Ni}, \mathrm{Co}$ content $(x)$. All Curie temperatures, determined from the inflection point of those curves, are above room temperature.

From the analysis of the major hysteresis loops (inset of Fig. 1), no information can be extracted about the interactions between the phases. This is also true for all the composites studied in this work.

Figure 3 shows the FORC distribution of each of the individual phases (alloys with $x=8.25$ and $x=11$ ), together with the FORC distribution of the composite material that corresponds to the magnetization curves presented in Fig. 1. All pure phase alloys FORC results are characterized by a single distribution of small coercivity (ranging between 0.1 and 0.3 Oe for the different alloys) [see, for example, Figs. 3 (a) and 3(b)], ascribing for a unique irreversible magnetization reversal process. The small FORC distribution enlargement, both along the interaction $\left(H_{u}\right)$ and coercive $\left(H_{c}\right)$ field axes, could be ascribed to some minor inhomogeneities in the magnetic properties of the amorphous ribbons. Composite FORC results, on the other hand, exhibit a more complex distribution shape [Fig. 3(c)]. A visual comparison between the individual phases and resulting composite FORC distributions already indicates that there are features that are not emerging from the algebraic addition of the two distributions of the individual alloys. Instead of a single distribution, the convolution of several distributions is present in composite FORC results, covering a larger field area than those of their respective alloys. In order to make a more quantitative comparison between the distributions and ascertain the contribution emerging from the interactions between phases, we have calculated the difference between the composite FORC distribution and the weighted average of the individual distributions of the phases (Fig. 4). Two precautions have to be taken in order to perform this procedure appropriately. The first one is that exactly the same ribbon pieces used for measuring the FORC curves of the individual 

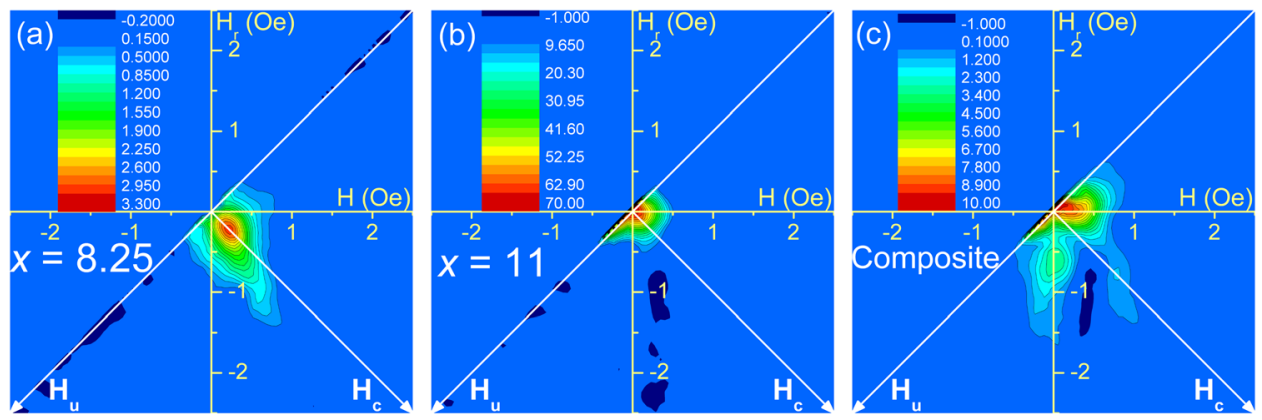

FIG. 3. Typical FORC diagrams of two individual phases $(x=8.25$ (a) and $x=11$ (b)) and of the composite constituted by them (c). The contour plot scale ranges from 0 (blue) to the FORC distribution maximum (red), while dark blue regions denote negative distributions.

phases were used to fabricate the layered composite material, maintaining exactly the same experimental conditions. Therefore, the contribution of each phase to the composite total distribution should remain the same (except for the effects arising from the interactions). The second precaution is related to the normalization of the distributions before performing the subtraction. It can be appropriately done in the following way:

$$
\begin{aligned}
\rho_{\text {difference }}\left(H_{r}, H\right)= & \frac{\rho_{\text {composite }}\left(H_{r}, H\right)}{\iint \rho_{\text {composite }}\left(H_{r}, H\right) d H_{r} d H} \\
& -\frac{1}{2}\left[\frac{\rho_{\text {phase } 1}\left(H_{r}, H\right)}{\iint \rho_{\text {phase } 1}\left(H_{r}, H\right) d H_{r} d H}\right. \\
& \left.+\frac{\rho_{\text {phase } 2}\left(H_{r}, H\right)}{\iint \rho_{\text {phase } 2}\left(H_{r}, H\right) d H_{r} d H}\right] .
\end{aligned}
$$

Without interaction between the phases, the FORC distribution difference would be expected to be completely null. However, all results exhibit a similar pattern: a centered negative region (in blue) surrounded by three positive regions

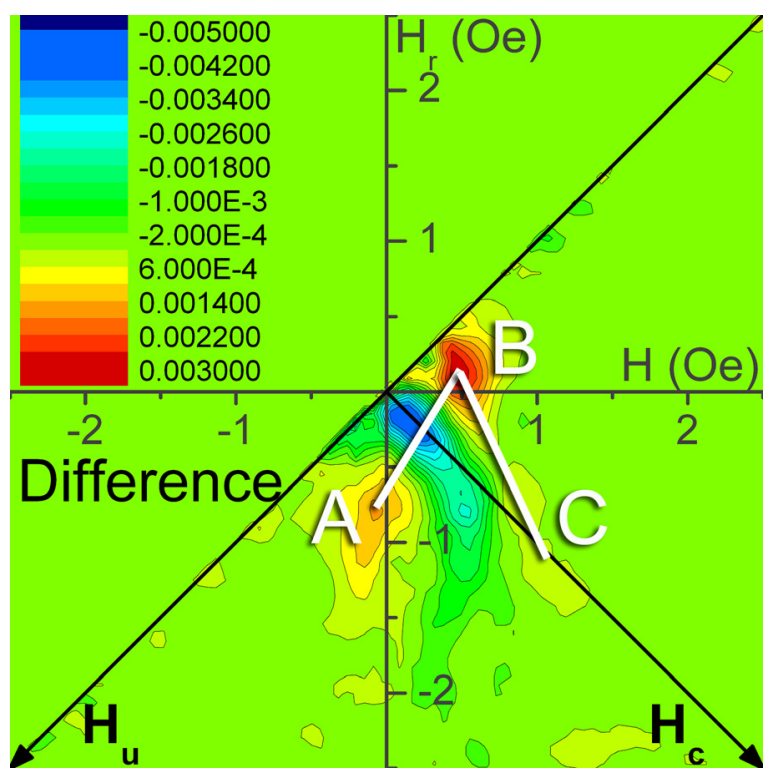

FIG. 4. Typical difference between the measured composite FORC distribution and the weighted average of those from individual phases, calculated according to Eq. (2). ( $x=8.25$ and 11 case represented). Maximum (positive) and minimum (negative) regions appear, respectively, in red and blue, while a null difference is represented in green. The "wishbone" pattern is marked with white lines. (marked A, B, and C in Fig. 4), therefore created by the phases interaction. Since negative regions account for hysterons predominantly present in the individual alloys, it is normal that they arise at the alloys FORC distribution position, i.e., low $H_{c}$ values and null $H_{u}$. Under interaction effect, those hysterons migrated mainly to higher $\left|H_{u}\right|$ values, giving rise to two maxima located at positions $\mathrm{A}$ and $\mathrm{B}$ on Fig. 4. They have roughly symmetric positions (with respect to the $H_{c}=0$ axis) along the direction of the $H_{u}$ axis and shifted along the positive direction of the $H_{c}$ axis. There is also a secondary migration of the hysterons to higher $H_{c}$ axis while still on it (C peak on Fig. 4). Linking together the peaks results in a well-known FORC pattern called wishbone (emphasized by the white solid line on Fig. 4). ${ }^{13}$ It arises when a narrow coercivity distribution is affected by a larger demagnetizing interaction field, usually directly proportional to the magnetization. The lack of clear FORC distribution delimitation prevents us to extract a precise interaction field value, but a good estimate can be taken as half the distance between peaks A and B. ${ }^{14,15}$ All composite samples present a qualitatively similar behavior, with interaction fields of $1.1 \pm 0.2$ Oe.

There is a remarkable difference in the magnitude of the interaction field determined using the magnetization FORC method and the one that was previously determined from the analysis of the magnetocaloric effect, ${ }^{6}$ which was around 4000 Oe for a composite formed by the alloys with $x=8.25$ and $x=11$, made from the same pieces of ribbons. The reason for this discrepancy should be ascribed to the different features that are detected with each technique. The magnetocaloric response of a material increases with magnetic field as a power law, ${ }^{16}$ therefore making this method more sensitive to large fields. On the contrary, FORC distributions can only be non-null for field values below the irreversibility limit of the alloy, i.e., inside the hysteresis loop, making this technique more sensitive to lower fields. Taking into account that all the alloys exhibit irreversible behavior only up to $2 \mathrm{Oe}$, it is natural that the interaction fields detected are below this value. However, it is worth noticing that the interaction field is still one order of magnitude larger than coercivity. We should not fully disregard the minor influence of the different shapes of the samples used in both techniques: long pieces of ribbon for FORC measurements and small alloy pieces for MCE, which could slightly alter the FORC distributions due to differences in the demagnetizing field. However, these changes would not alter the conclusions of this study. 
In conclusion, it has been shown that FORC analysis clearly indicates the existence of interphase interactions in layered composite samples. The value of the interaction field is one order of magnitude larger than the coercivity of the composite, but it lies remarkably below the interaction field determined from the analysis of magnetocaloric results. This discrepancy can be associated to the different sensitivity of these experimental methods: FORC technique is only sensitive to interaction fields whose magnitude lies within the hysteretic region of the magnetization curves, while the magnetocaloric response is more sensitive to larger fields. Moreover, FORC curves account for the irreversibility of the magnetization curves, while MCE focuses on a reversible effect. Therefore, care has to be taken when comparing results emerging from these intrinsically different experimental techniques.

This work was supported by the Science Without Borders Program of the Brazilian funding agency CNPq (\#401921/2013-1), the Spanish MINECO and EU FEDER (Project No. MAT 2013-45165-P) and the PAI of the Regional Government of Andalucía (Project No. P10-FQM6462).
${ }^{1}$ A. Rowe, Int. J. Refrig. 34, 168 (2011).

${ }^{2}$ V. Franco, J. S. Blázquez, B. Ingale, and A. Conde, Annu. Rev. Mater. Res. 42, 305 (2012).

${ }^{3}$ A. Smaili and R. Chahine, J. Appl. Phys. 81, 824 (1997).

${ }^{4}$ R. Caballero-Flores, V. Franco, A. Conde, K. E. Knipling, and M. A. Willard, Appl. Phys. Lett. 98, 102505 (2011).

${ }^{5}$ S. C. Paticopoulos, R. Caballero-Flores, V. Franco, J. S. Blazquez, A. Conde, K. E. Knipling, and M. A. Willard, Solid State Commun. 152, 1590 (2012).

${ }^{6}$ C. Romero-Muniz, V. Franco, and A. Conde, Appl. Phys. Lett. 102, 082402 (2013).

${ }^{7}$ F. Béron, D. Ménard, and A. Yelon, J. Appl. Phys. 103, 07D908 (2008).

${ }^{8}$ I. D. Mayergoyz, IEEE Trans. Magn. 22, 603 (1986).

${ }^{9}$ C. R. Pike, A. P. Roberts, and K. L. Verosub, J. Appl. Phys. 85, 6660 (1999).

${ }^{10}$ K. E. Knipling, M. Daniil, and M. A. Willard, Appl. Phys. Lett. 95, 222516 (2009).

${ }^{11}$ F. Béron, G. Soares, and K. R. Pirota, Rev. Sci. Instrum. 82, 063904 (2011).

${ }^{12}$ L. Clime, A. Stancu, P. Ciureanu, and A. Yelon, J. Optoelectron. Adv. Mater. 6, 1005 (2004).

${ }^{13}$ C. R. Pike, C. A. Ross, R. T. Scalettar, and G. Zimanyi, Phys. Rev. B 71, 134407 (2005).

${ }^{14}$ F. Béron, L. Clime, M. Ciureanu, D. Ménard, R. W. Cochrane, and A. Yelon, J. Nanosci. Nanotechnol. 8, 2944 (2008).

${ }^{15}$ F. Béron, L. A. S. de Oliveira, M. Knobel, and K. R. Pirota, J. Phys. D: Appl. Phys. 46, 045003 (2013).

${ }^{16}$ V. Franco and A. Conde, Int. J. Refrig. 33, 465 (2010). 